\title{
Modeling Correlation Structure for Collateralized Debt Obligations
}

\author{
Deniz Ilalan ${ }^{1}$ \\ ${ }^{1}$ Department of Banking and Finance, Cankaya University, Ankara, Turkey \\ Correspondence: Deniz Ilalan, Doctor of Financial Mathematics, Department of Banking and Finance, Cankaya \\ University, Eskisehir Yolu 29. Km, Ankara, 06810, Turkey. E-mail: denizilalan@cankaya.edu.tr
}

Received: February 12, 2015

Accepted: March 2, $2015 \quad$ Online Published: March 31, 2015

doi:10.5430/ijfr.v6n2p72

URL: http://dx.doi.org/10.5430/ijfr.v6n2p72

\begin{abstract}
Pricing complex financial derivatives such as collateralized debt obligations (CDO) is considered as the main reason triggering the 2008 financial crisis. The correlation structure related to the credit risks involved in a portfolio for pricing issues have been tried to overcome via a Gaussian copula framework first introduced by David Li (2000). This approach regards the correlation among the credit risks as normally distributed (tied with a Gaussian copula framework), enabling us to derive analytical solutions. However, despite its simplicity, this approach is far from reality, which caused mispricing of the tranches of CDOs. This phenomenon is called the correlation smile. This paper takes the correlation smile issue by considering a Levy copula framework. When this is introduced to pricing equations, one can see that the correlation smile is "corrected". Thus, a more accurate model of pricing the above-mentioned tranches is introduced.
\end{abstract}

Keywords: collateralized debt obligation (CDO), Gaussian copula, Levy copula

\section{Introduction}

Credit derivatives market drew quite a lot of attention beginning from 1998 and experienced a vast growth until the financial market crises in 2008. The asset price bubble in 2008 caused a shrinkage, however, could not be able to eliminate huge trading amounts of these products.

Typically, a credit derivative is a financial asset which takes into account the possibility of the default of a certain underlying instrument. A widely used credit derivative is a credit default swap (CDS). In a CDS agreement, the protection buyer is insured against the default of an asset backed loan via paying periodic predetermined installments to the protection seller as in an ordinary insurance contract.

On the other hand, a collateralized debt obligation (CDO) is another extensively used credit derivative which is usually regarded as the main instrument triggering the 2008 world financial crisis. CDO is a portfolio of credit derivatives which consist of multiple tranches, each having a different risk and return profile. The structure of a CDO is as follows: Regional banks assign certain loans to public usually collateralized with mortgage. These loans are paid with periodic predetermined installments. An investment bank acts as a gatherer of these loans in a pool. When these loans are collected, the investment bank arranges certain tranches as senior, mezzanine and junior (equity). Whenever the pool is filled with payments, the senior tranche is firstly paid, then mezzanine and finally the junior tranche gets paid. In that sense, junior tranche is the most vulnerable among them. If defaults happen there will be no flow of payment from the loan pool to the junior tranche. Thus junior tranche is attractive to risk lover investors. Since more risk means more return, the junior trance owners are promised to be paid higher return than other tranches. The return structure gradually decreases from junior to senior tranches. The investment banks were rated by the trusted rating agencies where even one day prior to sub prime meltdown, Lehman Brothers was rated top by three big rating agencies namely Standards \& Poors, Fitch and Moody's. Although these ratings put Lehman Brothers to a position of "as reliable as the US government", still the senior tranches were paying interest which was considerably above the government T-bill rates. This was one of the attractiveness of these instruments.

At the beginning, the credit pools were consisting of actual loans given to public. The loans were basically mortgage based loans, credit cards etc. Later on the structure became a Ponzi scheme. Since the investment banks were top rated, there was no cut in the demand for CDO tranches. Therefore, in order to meet higher demands, banks began offer loans to low paying ability customers. This rendered the quality of the loan pool to diminish, thus the number of defaults to increase. 
Meanwhile, the banks secured themselves via taking collaterals as houses for mortgage based loans. Up to sub prime meltdown the real estate prices perform a steady growth for nearly a decade; therefore the banks safely gave those loans to low paying ability clients. The sub prime meltdown not only raised questions regarding the credibility of investment banks it also seriously undermined the reputation of credit rating agencies and FED. After the collapse, in order to boost up the economy, FED decided to follow some unorthodox monetary policies as quantitative easing. As a butterfly effect major banks through the world began to pump money into economies. However, this did not go as expected, since the newly created money flows to financial markets instead of real economies. Nowadays, we are still facing the problem of excess liquidity and decreasing growth in the entire world. Yet, after these loose monetary policies we began to witness new records in stock exchanges which render the trading of these complex derivatives to increase even further.

In addition to all these, there was another quite important issue which was not taken seriously by the investment banks due to possible computation complexities. The pricing of these CDOs is not obvious in the sense that the expected time of defaults of the loan payers has to be taken into account. Moreover, it is known that due to recessions or depressions the default correlation among different sectors in the economy tends to rise. A typical CDO consists of 125 loans, so in order to calculate the price of this $\mathrm{CDO}$ we have to know the correlation structure of these loans which corresponds to a variance covariance matrix of 7750 cells!

It was the first attempt introduced by David Li (2000) that the correlation structure can be represented by a single common factor " $a$ " yielding a single factor Gaussian copula framework enabling the practitioners to compute the price of these complex financial derivatives. However, this computation technique inherited a major drawback which came to surface at the financial crisis.

The Gaussian copula approach rendered the tranches to be mispriced, thus triggering some inefficiency in the market. Although several attempts are proposed to overcome this issue, practitioners still use this technique just as they do to compute the prices of options via Black-Scholes model keeping mind the volatility smile phenomenon. Here, we face with another smile surface, namely the correlation smile. The smile shape comes from the implied correlation paramater derived from the existing tranche spreads in the market. The methodology of treating the tranches in a separate manner also exhibits a uniqueness of solution problem. Later, a base correlation approach is introduced which takes into account the pricing of a series several tranches in order to fit the correlation skew curve. This paper, contorary to the literature, tries to understand the effect of unexpected jumps in the pricing of CDO tranches by the introduction of a Levy copula which directly intervenes into the pricing formula.

The remaining of the paper is as follows: In the next section, Gaussian copula which is presented by David Li is summarized in the sense that it is the benchmark for pricing these complex derivatives throughout the literature. Therefore, in order to grasp the idea of $\mathrm{Li}$, basic preliminaries regarding copulas are given. Then, the major drawback of this pricing technique is considered. In Section 3 Levy copulas are introduced and the new model is discussed. Section 4 is devoted to numerical results and comparisons. Finally Section 5 concludes.

\section{Pricing of the CDO Tranches via Gaussian Copula Framework}

Let $X$ and $Y$ be random variables with distribution functions $F(x)=P[X \leq x], G(y)=P[Y \leq y]$ and joint distribution function $H(x, y)=P[X \leq x, Y \leq y]$. For each pair of real numbers $(x, y)$ three numbers can be associated, namely $F(x), G(y)$ and $H(x, y)$ each lying on the interval [0,1]. In other words, each pair $(x, y)$ gives a point $(F(x), G(y))$ in the unit square $[0,1] \times[0,1]$, and this ordered pair corresponds to a number $H(x, y)$ in $[0,1]$. This correspondence, which assigns the value of the joint distribution function to each ordered pair of values of individual functions, is called a copula.

Let $H$ be a joint distribution function with boundaries $F$ and $G$. Then there exists a copula $C$ such that for all $x, y$ in $\mathbb{R}$. If $F$ and $G$ are continuous, then $C$ is unique; otherwise, $C$ is uniquely determined on $\operatorname{Ran} F \times \operatorname{Ran} G$. Conversely, if $C$ is a copula and $F$ and $G$ are distribution functions, then the function $H$ defined by $H(x, y)=C(F(x), G(y))$ is a joint distribution function with margins $F$ and $G$. This is known as the Sklar's theorem.

Let us consider a synthetic CDO, a CDO formed by CDSs. A protection seller of a synthetic CDO obtains intermittent payments from the protection buyer, acting as a security for certain losses of subordinated tranches. The loss that the protection seller has to reimburse is determined via a reference credit portfolio. 
The pricing of a synthetic CDO works similar as the pricing of ordinary CDSs since a synthetic CDO consists of CDSs. To calculate the loss of a certain tranche say from $K_{1}$ to $K_{2}$ (with $0 \leq K_{1}<K_{2} \leq 1$ ) of the reference portfolio let us make further assumptions and introduce some new notations.

Assume that the spread payment dates are discrete, $\mathrm{t}_{1}<\mathrm{t}_{2}<\ldots<\mathrm{t}_{\mathrm{n}}=T$ where $T$ denotes the maturity of the synthetic CDO. Actually, this makes quite sense since the payment due dates are also discrete in real life. Some further relevant notations are as follows:

- $s$ denotes the yearly spread payments made by the protection buyer

- $\quad L_{K_{1} K_{2}}^{R}(t)$ denotes the loss of tranche $K_{1}, K_{2}$ up to time $t$, taking into consideration the possibility of recoveries. Usually models assume zero recovery, which is also the assumption; however, conventionally recovery rate is taken as $40 \%$. Moreover, there are models taking into account stochastic recovery rates which are beyond the aim of this work.

- The short term interest rate $r(t)$ is given, considered to be constant and independent of the tranche loss. Again, there are studies taking into account stochastic interest rate which according to us is not the main item in pricing synthetic CDOs.

Now, utilizing from Girsanov's theorem, consider the risk neutral measure and denote it by Q. Denote the expectation of the abovementioned tranche loss under this new measure by $\mathrm{E}_{\mathrm{Q}}\left[L_{K_{1}, K_{2}}^{R}(t)\right]$ or simply by $E L_{K_{1}, K_{2}}^{R}(t)$. The discount factor is $\mathrm{E}_{\mathrm{Q}}\left[\exp \left(-\int_{t_{0}}^{t_{i}} r(u) d u\right)\right]$ or simply $D\left(t_{0}, t_{i}\right)$.

The valuation of a synthetic CDO very much looks like the valuation of a simple swap. One first has to evaluate the present value of the spread payments, then evaluate the discounted value of protection payments taking into account the expectation of defaults, and finally equalize them.

The value of the premium leg is computed as follows:

$$
\begin{aligned}
\text { Premium Leg } & =\sum_{\mathrm{i}=1}^{\mathrm{n}} \Delta t_{i} \cdot s \cdot \mathrm{E}_{\mathrm{Q}}\left[\left(1-L_{K_{1}, K_{2}}^{R}\left(t_{i}\right)\right) e^{-\int_{t_{0}}^{t_{i}} r(u) d u}\right] \\
& =\sum_{\mathrm{i}=1}^{\mathrm{n}} \Delta t_{i} \cdot s \cdot\left[\left(1-E L_{K_{1}, K_{2}}^{R}\left(t_{i}\right)\right) \cdot D\left(t_{0}, t_{i}\right)\right],
\end{aligned}
$$

where $\Delta t_{i}=t_{i}-t_{i-1}$.

Protection payments are made in case of a default. For simplicity, integration is avoided by discretization. Therefore we have the following:

$$
\begin{aligned}
\text { Protection Leg }= & \mathrm{E}_{\mathrm{Q}}\left[\int_{t_{0}}^{t_{n}} e^{-\int_{t_{0}}^{s} r(u) d u} d L_{K_{1}, K_{2}}^{R}(s)\right] \\
& \approx \sum_{\mathrm{i}=1}^{\mathrm{n}} \mathrm{E}_{\mathrm{Q}}\left[e^{-\int_{t_{0}}^{t_{i}} r(u) d u}\left(L_{K_{1}, K_{2}}^{R}\left(t_{i}\right)-L_{K_{1}, K_{2}}^{R}\left(t_{i-1}\right)\right)\right] \\
& =\sum_{\mathrm{i}=1}^{\mathrm{n}} \mathrm{E}_{\mathrm{Q}}\left(E L_{K_{1}, K_{2}}^{R}\left(t_{i}\right)-E L_{K_{1}, K_{2}}^{R}\left(t_{i-1}\right)\right) \cdot D\left(t_{0}, t_{i}\right) .
\end{aligned}
$$


Equalizing the premium and protection leg yields:

$$
s=\frac{\sum_{\mathrm{i}=1}^{\mathrm{n}} \mathrm{E}_{\mathrm{Q}}\left(E L_{K_{1}, K_{2}}^{R}\left(t_{i}\right)-E L_{K_{1}, K_{2}}^{R}\left(t_{i-1}\right)\right) \cdot D\left(t_{0}, t_{i}\right)}{\sum_{\mathrm{i}=1}^{\mathrm{n}} \Delta t_{i} \cdot s \cdot\left[\left(1-E L_{K_{1}, K_{2}}^{R}\left(t_{i}\right)\right) \cdot D\left(t_{0}, t_{i}\right)\right]} .
$$

In case a loss occurs in the portfolio denoted by $L_{\text {porffolio }}(t)$, one can calculate the corresponding percentage loss as follows:

$$
L_{K_{1}, K_{2}}^{R}(t)=\frac{\min \left(\left(L_{\text {portfolio }}^{R}(t), K_{2}\right)-K_{1}\right)^{+}}{K_{2}-K_{1}} .
$$

If the discrete distribution of the aggregate loss of the reference portfolio up to time $t$ is known (considering recoveries) and can take only $m$ possible values then,

$$
\mathrm{L}_{\text {portfolio }}^{\mathrm{R}}(\mathrm{t})=\left\{\mathrm{L}_{\text {portfolio }}^{\mathrm{R}, \mathrm{k}}(\mathrm{t}) \text { with risk neutral probability } \mathrm{F}^{\mathrm{R}}(\mathrm{t}, \mathrm{k})\right\}_{\mathrm{k}=1, \ldots, \mathrm{m}}
$$

Thus, we have the following:

$$
\begin{aligned}
& E L_{K_{1}, K_{2}}^{R}(t)=E_{Q}\left[\frac{\min \left(\left(L_{\text {portfolio }}^{R}(t), K_{2}\right)-K_{1}\right)^{+}}{K_{2}-K_{1}}\right] \\
& =\frac{1}{K_{2}-K_{1}} \sum_{k=1}^{m} \min \left(\left(L_{\text {portfolio }}^{R}(t), K_{2}\right)-K_{1}\right)^{+} \cdot F^{R}(t, k) .
\end{aligned}
$$

If the loss distribution function of the portfolio is continuous, then

$$
\begin{aligned}
E L_{K_{1}, K_{2}}^{R}(t) & =\frac{1}{K_{2}-K_{1}}\left(\int_{K_{1}}^{1}\left(\min \left(x, K_{2}\right)-K_{1}\right) d F^{R}(t, x)\right) \\
& =\frac{1}{K_{2}-K_{1}}\left(\int _ { K _ { 1 } } ^ { K _ { 2 } } \left(\left(x-K_{1}\right) d F^{R}(t, x)+\int_{K_{2}}^{1}\left(\left(K_{2}-K_{1}\right) d F^{R}(t, x)\right)\right.\right. \\
& =\frac{1}{K_{2}-K_{1}}\left(\int _ { K _ { 1 } } ^ { 1 } \left(\left(x-K_{1}\right) d F^{R}(t, x)-\int_{K_{2}}^{1}\left(\left(x-K_{1}\right) d F^{R}(t, x)+\int_{K_{2}}^{1}\left(\left(K_{2}-K_{1}\right) d F^{R}(t, x)\right)\right.\right.\right. \\
& =\frac{1}{K_{2}-K_{1}}\left(\int _ { K _ { 1 } } ^ { 1 } \left(\left(x-K_{1}\right) d F^{R}(t, x)-\int_{K_{2}}^{1}\left(\left(x-K_{2}\right) d F^{R}(t, x)\right) .\right.\right.
\end{aligned}
$$

It can realized that it is crucial in the pricing of a CDO tranche to obtain the loss distribution function. The benchmark model for this, which will be presented below is the one factor Gaussian copula model first introduced by David Li.

Let $\tau_{i}$ be a random variable denoting the time to default of a firm $i$ from the reference portfolio. Instead of concentrating the firms default times one by one, under this framework the default correlations among the firms become crucial for pricing. The Gaussian copula approach allows us to price CDO tranches without taking into account the marginal distribution of the firms. 
Let $A_{i}(t)$ be the standardized asset return of firm $i$ up to time which is assumed to be of the following form:

$$
A_{i}(t)=a_{i} M(t)+\sqrt{1-a_{i}^{2}} X_{i}(t)
$$

where $M(t)$ is the market risk and $X_{i}(t)$ is the firm based (idiosyncratic) risk, both standard normally distributed. (This is a commonly used convention since asset returns are "almost" normally distributed as the Black-Scholes option pricing formula proclaims). The above equation renders $A_{i}(t)$ to be standard normally distributed as well. If we denote the distribution function of the default time $\tau_{i}$ by $Q_{i}$ then the issuer said to be defaulted before time $t$ when $\Phi\left(A_{i}(t)\right) \leq Q_{i}(t)$ or equivalently $A_{i}(t) \leq \Phi^{-1}\left(Q_{i}(t)\right)=: C_{i}(t)$. Here $Q_{i}(t)=Q\left[\tau_{i} \leq t\right]$ where $Q$ denotes the risk neutral probability implied from the observable market prices. In fact, as we shall see later, in the Gaussian copula framework the whole asset return structure of the firms reduce to a single component, which is derived from the existing market prices, enabling us to price the tranches of CDOs. According to the asset return equation the $i^{\text {th }}$ issuer defaults up to time $t$ when

$$
X_{i}(t) \leq \frac{C_{i}(t)-a_{i} M(t)}{\sqrt{1-a_{i}^{2}}},
$$

Then the probability that the $i^{t h}$ issuer defaults up to time $t$, conditional on the factor $M(t)$ becomes,

$$
p_{i}(t \mid M) \leq \Phi\left(\frac{C_{i}(t)-a_{i} M(t)}{\sqrt{1-a_{i}^{2}}}\right) .
$$

Just like the idea of central limit theorem, here the aim is to derive analytical results for pricing the CDO tranches for the limiting cases. The related assumptions for the credit issuers now are:

- same portfolio weights

- $\quad$ same default probability $Q(t)$

- $\quad$ same recovery rate $R$

- same correlation to the sensitivity of the market

Then we have

$$
p(t \mid M) \leq \Phi\left(\frac{C(t)-a M(t)}{\sqrt{1-a^{2}}}\right) .
$$

In large homogeneous portfolio model, the expected loss at time $t$ of the mezzanine tranche taking losses from $K_{1}$ to $K_{2}$ percent of the overall portfolio assuming zero recovery is given by

$$
E L_{K_{1}, K_{2}}(t)=\frac{\Phi_{2}\left(-\Phi^{-1}\left(K_{1}, C(t), \rho\right)\right)-\Phi_{2}\left(-\Phi^{-1}\left(K_{2}, C(t), \rho\right)\right)}{K_{2}-K_{1}},
$$

where $\Phi_{2}$ is the bivariate normal distribution function with the covariance matrix

$$
\rho=\left(\begin{array}{cc}
1 & -\sqrt{1-a^{2}} \\
-\sqrt{1-a^{2}} & 1
\end{array}\right) .
$$




\section{Levy Copulas}

A stochastic process $\left(X_{t}\right)$ on $\mathbb{R}^{d}$ with $X_{0}=0$ is called a Levy process if:

- For $n \geq 1$ and $0 \leq t_{0}<t_{1}<\ldots<t_{n} \leq T$, the random variables $X_{t_{0}}, X_{t_{1}}-X_{t_{0}}, X_{t_{2}}-X_{t_{1}}, \ldots, X_{t_{n}}-X_{t_{n-1}}$ are independent

- The distribution of $X_{t+s}-X_{t}$ does not depend on $t$

- For every $t \in[0, T]$ and $\in>0, \lim _{s \rightarrow t} P\left[\left|X_{s}-X_{t}\right|>\varepsilon\right]=0$

- $\exists \Omega_{0} \in F$ with $P\left[\Omega_{0}\right]=1$ such that $\forall \omega \in \Omega_{0}, X_{t}(\omega)$ is right-continuous and has left limits in $t \in[0, T]$. This definition allows a Levy process $\left(X_{t}\right)$ to have discontinuities (jumps). The jump behavior is described by the jump measure $\mu$ defined on $[0, T] \times \mathbb{R}^{d}$ by $\mu(A)=\#\left\{\left(t, \Delta X_{t}\right) \in A\right\}$. For every measurable set $A \subset \mathbb{R}^{d}$, $\mu\left(\left[t_{1}, t_{2}\right] \times A\right)$ counts the number of jumps with sizes not exceeding $A$ between $t_{1}$ and $t_{2}$. On the other hand, Levy measure $v$ is defined as $v(A)=E\left[\#\left\{t \in[0,1]: \Delta X_{t} \neq 0, \Delta X_{t} \in A\right\}\right]$, indicating the expected number of jumps, per unit time belonging to $A$ where $A \in B\left(\mathbb{R}^{d}\right)$.

Let $\left(X_{t}\right)$ be a Levy process on $\mathbb{R}^{d}$ with Levy measure $v$. The tail integral of $v$ is a function $U:\left(\mathbb{R}^{d} \backslash\{0\}\right) \mapsto \mathbb{R}$ defined as follows

$$
U\left(x_{1}, \ldots, x_{d}\right)=v\left(\prod_{i=1}^{d} I\left(x_{i}\right)\right) \prod_{i=1}^{d} \operatorname{sgn}\left(x_{i}\right)
$$

where

$$
I(x)=\left\{\begin{array}{l}
{[x, \infty), x>0} \\
(-\infty, x], x<0 .
\end{array}\right.
$$

A function $F: \mathbb{R}_{\infty}^{d} \mapsto \mathbb{R}_{\infty}$ is called a Levy $d$-copula function (or Levy copula), if

- $\quad F\left(u_{1}, \ldots, u_{d}\right) \neq \infty$ for $\left(u_{1}, \ldots, u_{d}\right) \neq(\infty, \ldots, \infty)$

- $\quad F\left(u_{1}, \ldots, u_{d}\right)=0$ if $u_{i}=0$ for at least one $i \in\{1, \ldots, d\}$

- $\quad F$ is $d$-increasing

- $\quad F_{i}(u)=u$ for any $i \in\{1, \ldots, d\}, u \in \mathbb{R}$

Let $v$ be a Levy measure on $\mathbb{R}^{d}$. Then there exists a Levy copula $F$ such that the tail integrals of $v$ satisfy $U_{I}\left(\left(x_{i}\right)_{i \in I}\right)=F_{I}\left(\left(U_{i}\left(x_{i}\right)\right)_{i \in I}\right)$ for any non-empty $I \subset\{1, \ldots, d\}$ and any $\left(x_{i}\right)_{i \in I} \in \mathbb{R}^{I}$. Conversely, if $F$ is a $d$-dimensional Levy copula and $v_{1}, \ldots, v_{d}$ are Levy measures on $\mathbb{R}$ with tail integrals $U_{1}, \ldots, U_{d}$ then there exists a unique Levy measure $\mathbb{R}^{d}$ with one-dimensional tail integrals $U_{1}, \ldots, U_{d}$. This is known as the generalized Sklar's theorem.

Let $\left(X_{t}\right)$ be a Levy process on $\mathbb{R}^{d}$. Then the independence copula is given by

$$
F_{\perp}\left(u_{1}, \ldots, u_{d}\right)=\sum_{i=1}^{d} u_{i} \prod_{j \neq i} I_{\infty}\left(u_{j}\right)
$$

Let be a Levy process on $\mathbb{R}^{d}$ whose Levy measure is supported by an ordered set $D \subset S$. Then the complete positive dependence Levy copula is given by 


$$
F_{\|}\left(u_{1}, \ldots, u_{d}\right)=\min \left(\left|u_{1}\right|, \ldots,\left|u_{d}\right|\right) I_{S}+\left(u_{1}, \ldots, u_{d}\right) \prod_{i=1}^{d} \operatorname{sgn}\left(u_{i}\right)
$$

\section{Numerical Results and Comparison}

The Gaussian copula approach involves some improper integrals which has to be computed numerically. Moreover, the correlation coefficient " $a$ " involved in the variance covariance matrix should be calculated via an "implied correlation" from the existing market prices of CDO's. However, having obtained " $a$ " will only enable one to price the other tranches instantly. Here, the aim is to offer another theoretical model which explains the correlation surfaces better than the traditional approach. A direct application of Sklar's theorem yields

$$
f\left(x_{1}, x_{2}, \ldots, x_{n}\right)=\frac{\partial^{n}\left[C\left(F_{1}\left(x_{1}\right), \ldots, F_{n}\left(x_{n}\right)\right]\right.}{\partial F_{1}\left(x_{1}\right) \ldots \partial F_{n}\left(x_{n}\right)} \cdot \prod_{i=1}^{n} f_{i}\left(x_{i}\right)=c\left(F_{1}\left(x_{1}\right), \ldots, F_{n}\left(x_{n}\right)\right) \cdot \prod_{i=1}^{n} f_{i}\left(x_{i}\right)
$$

where $c\left(F_{1}\left(x_{1}\right), \ldots, F_{n}\left(x_{n}\right)\right)=f\left(x_{1}, x_{2}, \ldots, x_{n}\right) / \prod_{i=1}^{n} f_{i}\left(x_{i}\right)$

Let $\Theta$ be the parameter space and $\theta$ be the $k$-dimensional vector of parameters to be estimated. Let $L K L_{t}(\theta)$ and $l k l_{t}(\theta)$ be, respectively, the likelihood and log-likelihood function for the observation at time $t$. Define the log-likelihood function $\operatorname{lkl}(\theta)$ as the following:

$$
l k l(\theta)=\sum_{t=1}^{T} l k l_{t}(\theta)
$$

Taking into account the canonical form described above

$$
l k l(\theta)=\sum_{t=1}^{T} \ln c\left(F_{1}\left(x_{1}^{t}\right), \ldots, F_{N}\left(x_{N}^{t}\right)\right)+\sum_{t=1}^{T} \sum_{n=1}^{N} \ln f_{n}\left(x_{n}^{t}\right)
$$

the maximum likelihood estimator can be defined accordingly.

For the Gaussian copula let $\Theta=\left\{V: V \in \mathbb{R}^{N x N}\right\}$ denote the parameter space with $V$ being a symmetric and positive definite matrix. The application of canonical form yields:

$$
l k l^{\text {gaussian }}(\theta)=-\frac{T}{2} \ln |V|-\frac{1}{2} \sum_{t=1}^{T} \varsigma_{t}{ }^{\prime}\left(V^{-1}-I\right) \varsigma_{t}
$$

Assuming that the log likelihood function is differentiable we have the solution of the equation $\partial / \partial \theta=0$ defining a global maximum. Thus the log likelihood for the Gaussian copula becomes:

$$
\frac{\partial}{\partial V^{-1}} l k l^{\text {gaussian }}(\theta)=\frac{T}{2} V-\frac{1}{2} \sum_{t=1}^{T} \varsigma_{t}{ }^{\prime} \varsigma_{t} \Rightarrow \hat{V}=\frac{1}{T} \sum_{t=1}^{T} \varsigma_{t}{ }^{\prime} \varsigma_{t}
$$

Let $A$ and $B$ the attachment and detachment points respectively. If we denote the accumulated loss of the reference portfolio at time $t$ as $L(t)$ the loss of the tranche can be given as:

$$
L^{A, B}(t)=(L(t)-A) \mathrm{I}_{\{A, B\}} L(t)+(B-A) \mathrm{I}_{\left\{B, \sum_{i=1}^{n} N_{i}\right\}} L(t)
$$

Just like a regular swap analysis a fair price of a CDO tranche is given by 


$$
s_{A, B}^{*}=\frac{E^{*}\left[\int_{o}^{T} D(0, t) d L^{A, B}(t)\right]}{E^{*}\left[\alpha \sum_{i=1}^{n} D\left(0, t_{i}\right) \min \left\{\max \left[B-L\left(t_{i}\right), 0\right], B-A\right\}\right]}
$$

where $D$ denotes the discount factor, the numerator being the expected loss (default leg) and the denominator being the installments (premium leg).

The simulation done by MatLab is divided into 3 parts

- Generating default times via Gaussian copula by the help of log likelihood estimation

- Equalizing the default and premium legs

- Calculating the tranche spreads for different values of correlation and recovery rates

For 125 loan payers, 1 year of maturity and 1000 simulations we have Figure 1, Figure 2 and Figure 3 as

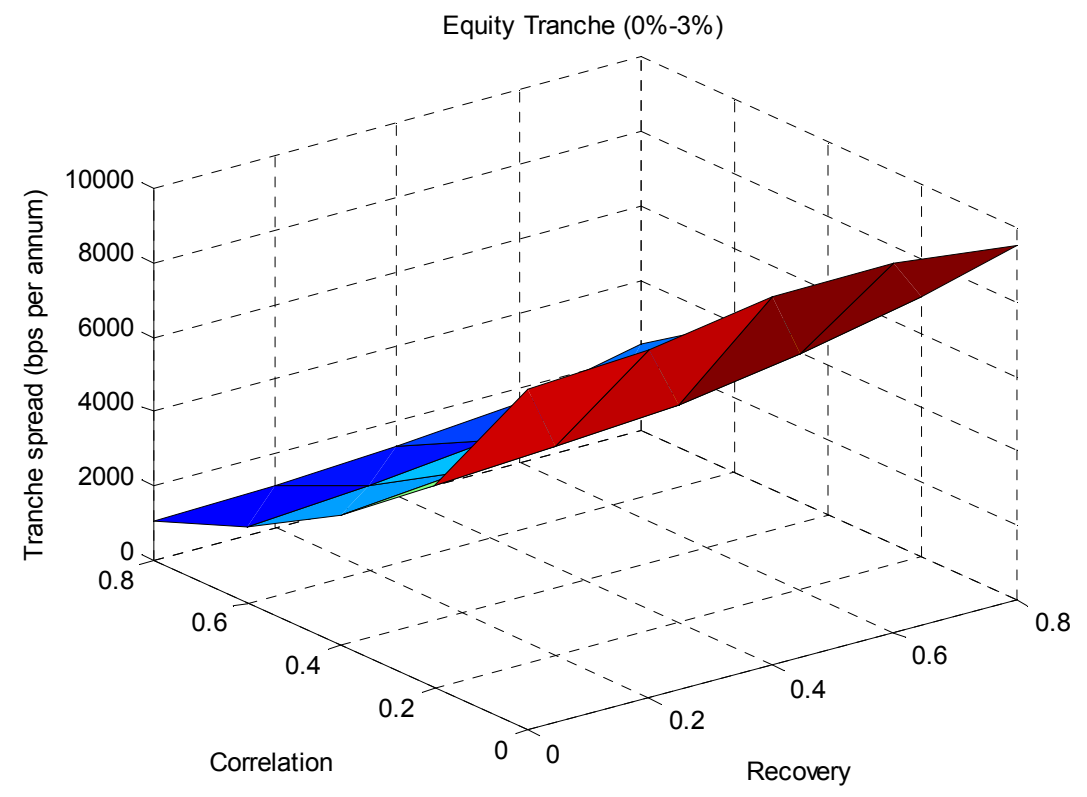

Figure 1. Equity tranche spread modeled with Gaussian copula for different recovery rates

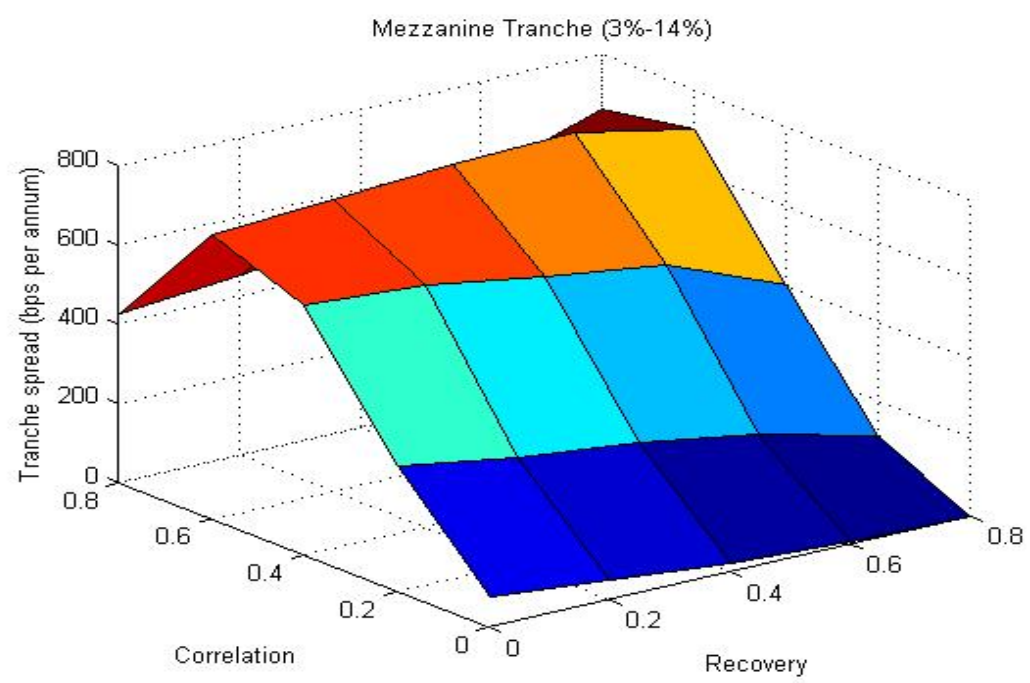

Figure 2. Mezzanine tranche spread modeled with Gaussian copula for different recovery rates 


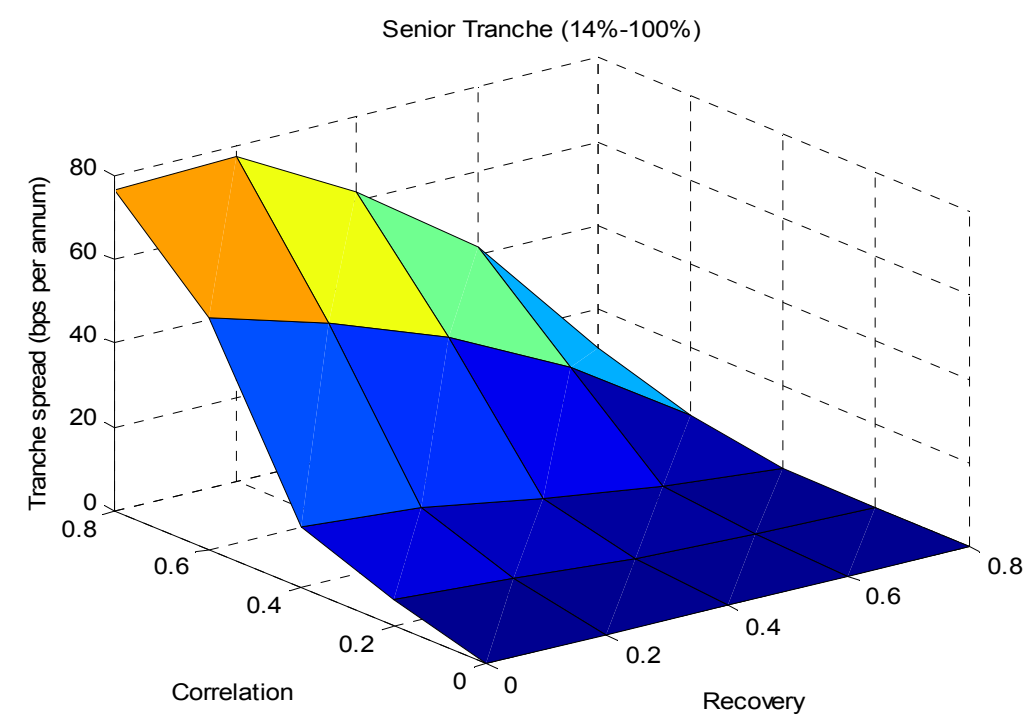

Figure 3. Senior tranche spread modeled with Gaussian copula for different recovery rates

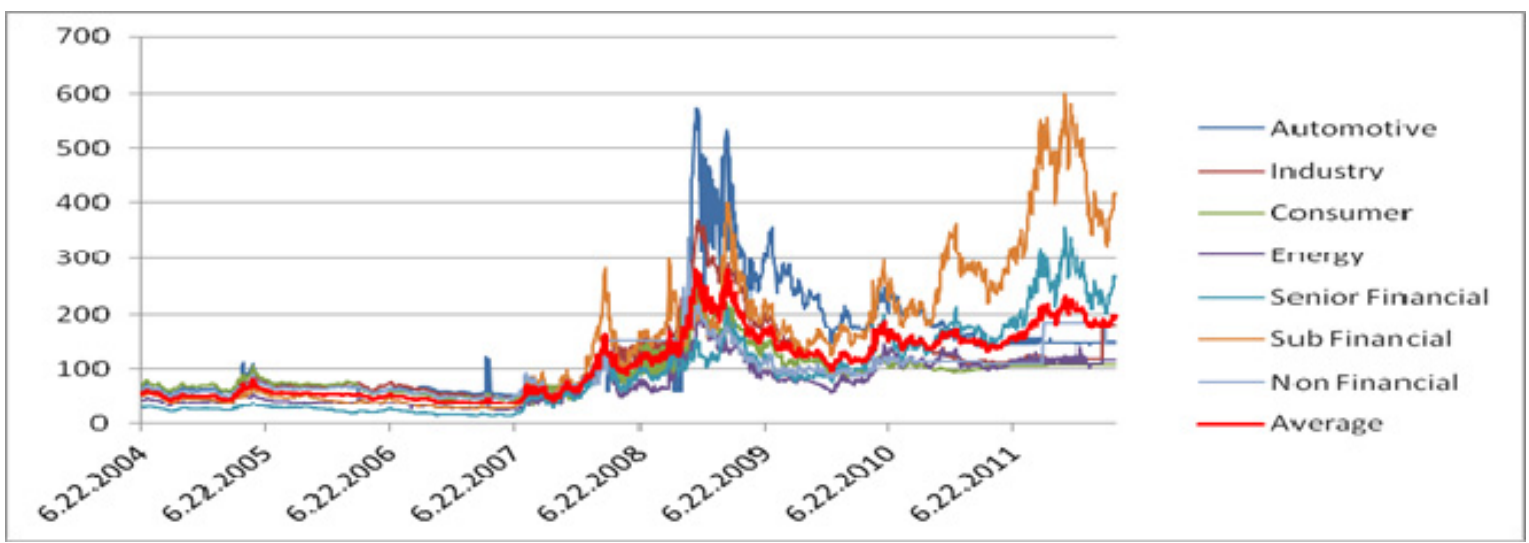

Figure 4. Itaxx125 CDS spreads for seven sectors and their average

The daily Itraxx125 data between 2004-2012 indicates that the 7 sectors on which the CDSs are written comprising the synthetic CDO have almost the same correlation structure during times of crises. That is, all sectors tend to jump at the same time. Actually this is not data specific in the sense that the CDS spreads have tendency to move together during crisis periods. For instance if there is a negative financial shock then we expect the possibility of bankruptcy to rise for all possible companies, even for the countries. Therefore for modeling purposes a perfectly dependent Levy copula having following form is added to the pricing

$$
F_{\|}=\min \left(\sum_{i=1}^{7}\left|u_{i}\right|\right) I_{S}+\left(u_{i}\right)_{i=1, \ldots, 7} \prod_{i=1}^{7} \operatorname{sgn}\left(u_{i}\right)
$$

Now again for 125 loan payers, 1 year of maturity and 1000 simulations, we now have Figure 5, Figure 6 and Figure 7 as 


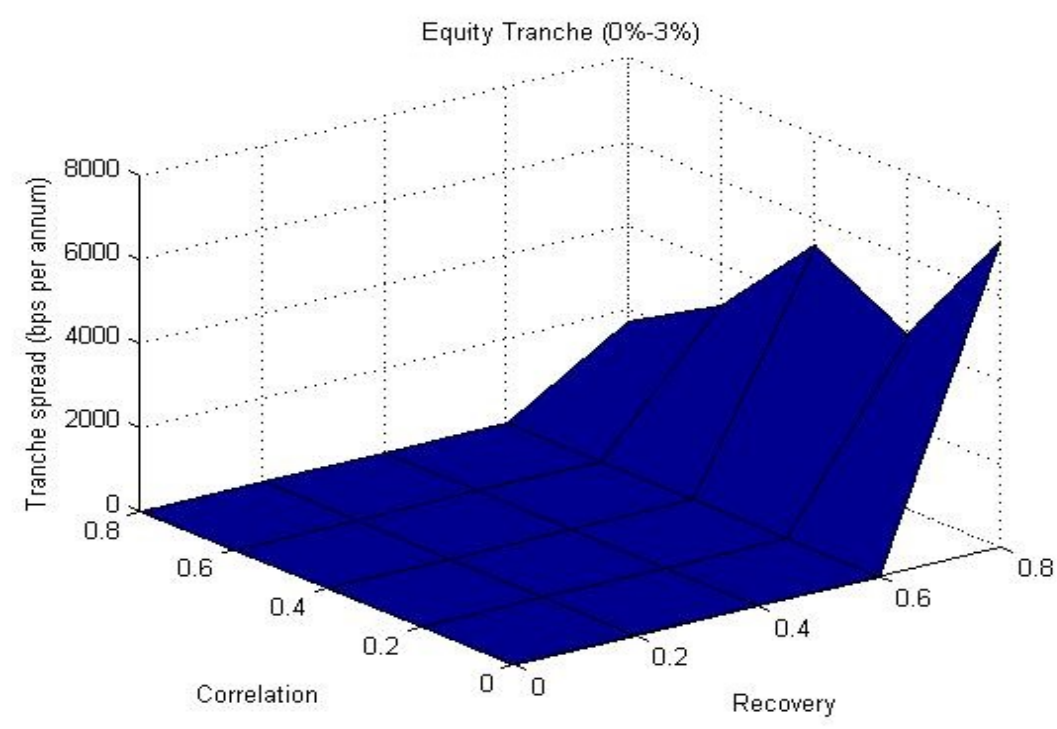

Figure 5. Equity tranche spread modeled with Levy copula for different recovery rates

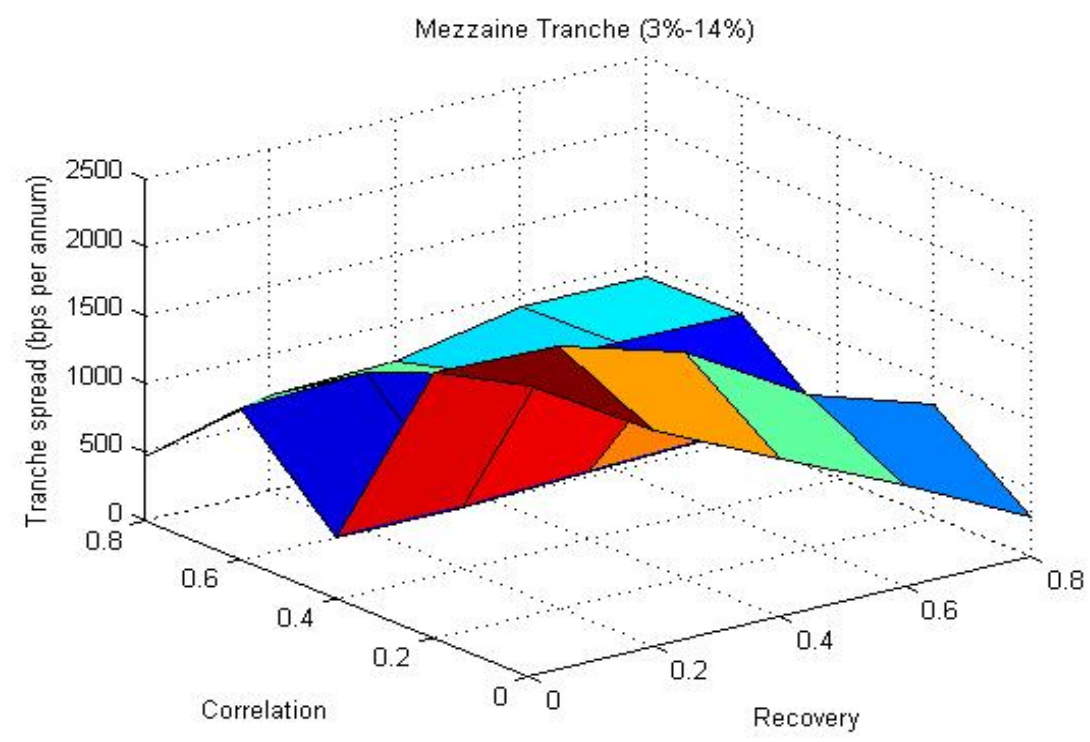

Figure 6. Equity tranche spread modeled with Levy copula for different recovery rates 


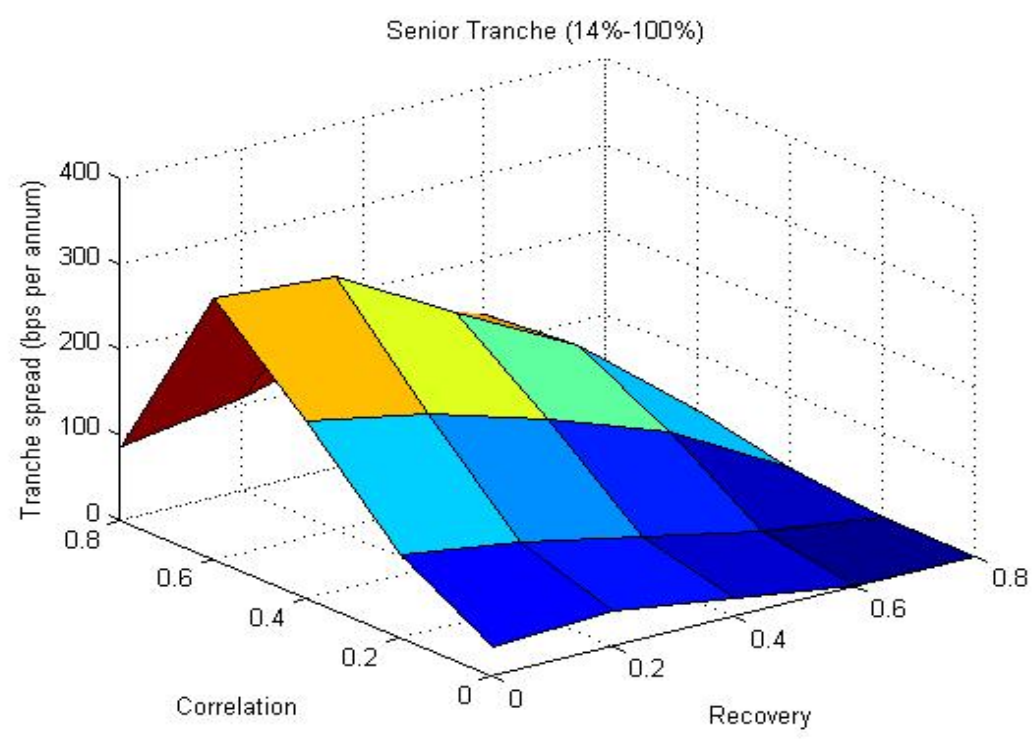

Figure 7. Equity tranche spread modeled with Levy copula for different recovery rates

Here the default times are generated by Gaussian copula conjoined with a Levy copula added to the variance covariance matrix as a "corrector".

\section{Summary and Conclusions}

With Gaussian copula framework we confront the "correlation smile" problem. This phenomenon occurs due to the inconsistency of single parameter correlation structure. When the correlation parameter is derived from the market prices i.e. implied correlation, senior tranches imply high correlation whereas junior and part of mezzanine tranches imply low correlation.

Comparing Figure 1 and Figure 5 we see that for junior tranche when correlation parameter gets higher so does the expected tranche loss and the associated fair spread. Gaussian copula framework implies higher correlation and thus higher fair spread. On the contrary the new model gives lower spread everywhere.

For senior tranche the case is different when we look at Figure 2 and Figure 6. Since senior tranche investors are affected only when losses in the collateral go beyond most of the pool notional, many defaults should occur in order for this to happen. However, in practice higher correlation implies lower spread since the probability of huge number of defaults is quite unlikely. Gaussian copula framework implies lower correlation and thus lower fair spread. The new model, on the other hand gives higher spread.

Finally, looking at Figure 3 and Figure 7, for mezzanine tranche, we witness that Gaussian copula model gives higher and sometime later lower correlation. The new model gives lower spread at the beginning and later higher spread which is consistent for correcting the correlation smile phenomenon.

\section{References}

Albrecher, H., Ladoucette, S.A., \& Schoutens, W. (2007). A Generic One-Factor Lévy Model for Pricing Synthetic CDOs. Advances in Mathematical Finance, Applied and Numerical Harmonic Analysis, 259-277.

Andersen L., \& Sidenius. J. (2004). Extensions to the Gaussian Copula: random recovery and random factor loadings. Journal of Credit Risk, 1(1).

Balakrishna, B. S. (2008). Levy Density Based Intensity Modeling of the Correlation Smile. Munich Personal RePEc Archive, MPRA Paper No. 14922.

Baxter, M. (2006). Levy Process Dynamic Modeling of Single-name Credits and CDO Tranches. Nomura Fixed Income Quant Group.

Bluhm, C., Overbeck, L., \& Wagner, C. (2003). An Introduction to Credit Risk Modeling. Chapman Hall/CRC, Financial Mathematics Series. 
Brigo, D., Pallavicini, A., \& Torresetti, R. (2010). Credit Models and The Crisis, A Journey into CDOs, Copulas, Correlations and Dynamic Models. Publisher: Wiley Finance.

Cont, R., \& Tankov, P. (2003). Financial Modeling with Jump Processes. Chapman and Hall/CRC Financial Mathematics Series.

Dorey, M., \& Joubert, P. (2005). Modeling Copulas: An Overview. The Staple Inn Actuarial Society.

Duffie, D. (2004). Time to adapt copula methods for modeling credit risk correlation. Risk, 77.

Duffie, D., \& Garleanu, N. (2001). Risk and valuation of collateralized debt obligations. Financial Analysts Journal, $57(1), 41-59$.

Feld, M. (2007). Implied Correlation Smile. Master's Thesis, Humboldt University Institute For Statistics and Econometrics.

Hull, J., \& White, A. (2004). Valuation of a CDO and an n-th to Default CDS without Monte Carlo Simulation. Journal of Derivatives, 12(2), 8-23. http://dx.doi.org/10.3905/jod.2004.450964

Hunting, M. (2007). Lévy processes and Lévy copulas with an Application in Insurance. Master's Thesis, University of Bergen, Mathematical Statistics.

Kallsen J., \& Tankov P. (2006). Characterization of dependence of multidimensional Lévy processes using Lévy copulas. Journal of Multivariate Analysis, 97(7), 1551-1572. http://dx.doi.org/10.1016/j.jmva.2005.11.001

Li, David X. (1998). Constructing a credit curve. Credit Risk, a Risk Special Report, 40-44.

Li, David X. (2000). On default correlation: A copula function approach. The Journal of Fixed Income, 9(4), 43-54. http://dx.doi.org/10.2139/ssrn.187289

London, J. (2006). Modeling Derivatives Applications in Mat Lab, C++, and Excel. Financial Times Press.

Meneguzzo, D., \& Vecchiato, W. (2004). Copula sensitivity in collateralized debt obligations and basket default swaps. The Journal of Futures Markets, 24(1), 37-70. http://dx.doi.org/10.1002/fut.10110

Nelsen, R., B. (2006). An Introduction to Copulas (2nd ed.). Springer. http://dx.doi.org/10.1007/0-387-28678-0

Schlosser, A. (2011). Pricing and Risk Management of Synthetic CDOs. Springer. http://dx.doi.org/10.1007/978-3-642-15609-0

Tavakoli, J. (2003). Collateralized Debt Obligations and Structured Finance: New Developments in Cash and Synthetic Securitization. Wiley Finance.

Torresetti, R., Brigo, D., \& Pallavicini, A. (2006). Implied Correlation in CDO tranches: a Paradigm to be handled with care. Credit Models, Banca IMI, Milan.

Trivedi, P.K., \& Zimmer, D.M. (2005). Copula Modeling: An Introduction for Practitioners. Foundations and Trends in Econometrics, 1(1).

Willemann, S. (2007). Fitting the CDO correlation skew: a tractable structural jump-diffusion model. Journal of Credit Risk, 3(1), 63-90. 